\title{
Comparison of Energy Efficiency and Power Density in Pressure Retarded Osmosis and Reverse Electrodialysis
}

\author{
Ngai Yin Yip and Menachem Elimelech* \\ Department of Chemical and Environmental Engineering, Yale University, New Haven, Connecticut 06520-8286, United States
}

Supporting Information

ABSTRACT: Pressure retarded osmosis (PRO) and reverse electrodialysis (RED) are emerging membrane-based technologies that can convert chemical energy in salinity gradients to useful work. The two processes have intrinsically different working principles: controlled mixing in PRO is achieved by water permeation across salt-rejecting membranes, whereas RED is driven by ion flux across charged membranes. This study compares the energy efficiency and power density performance of PRO and RED with simulated technologically available membranes for natural, anthropogenic, and engineered salinity gradients (seawater-river water, desalination

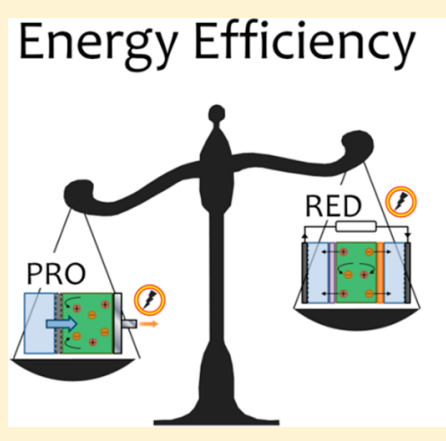
Power Density brine-wastewater, and synthetic hypersaline solutions, respectively). The analysis shows that PRO can achieve both greater efficiencies (54-56\%) and higher power densities (2.4-38 $\mathrm{W} / \mathrm{m}^{2}$ ) than RED $\left(18-38 \%\right.$ and $\left.0.77-1.2 \mathrm{~W} / \mathrm{m}^{2}\right)$. The superior efficiency is attributed to the ability of PRO membranes to more effectively utilize the salinity difference to drive water permeation and better suppress the detrimental leakage of salts. On the other hand, the low conductivity of currently available ion exchange membranes impedes RED ion flux and, thus, constrains the power density. Both technologies exhibit a trade-off between efficiency and power density: employing more permeable but less selective membranes can enhance the power density, but undesired entropy production due to uncontrolled mixing increases and some efficiency is sacrificed. When the concentration difference is increased (i.e., natural $\rightarrow$ anthropogenic $\rightarrow$ engineered salinity gradients), PRO osmotic pressure difference rises proportionally but not so for RED Nernst potential, which has logarithmic dependence on the solution concentration. Because of this inherently different characteristic, RED is unable to take advantage of larger salinity gradients, whereas PRO power density is considerably enhanced. Additionally, high solution concentrations suppress the Donnan exclusion effect of the charged RED membranes, severely reducing the permselectivity and diminishing the energy conversion efficiency. This study indicates that PRO is more suitable to extract energy from a range of salinity gradients, while significant advancements in ion exchange membranes are likely necessary for RED to be competitive with PRO.

\section{INTRODUCTION}

The Gibbs free energy from mixing two solutions of different concentration can be harnessed for useful work. ${ }^{1,2}$ The salinity gradient can be from various sources, ${ }^{2}$ such as the mixing of fresh river water with salty seawater, which occurs naturally as part of the hydrological cycle. A recent study showed that the $\sim 37300 \mathrm{~km}^{3}$ annual global river discharge represents a substantial source of clean and renewable energy that can potentially generate electricity for over half a billion people. ${ }^{3}$ Alternatively, anthropogenic waste streams can be utilized, e.g., concentrated brine from desalination plants can be paired with wastewater effluent from treatment facilities and the power generated can partially offset the desalination energy cost., ${ }^{4,5}$ Industries discharge approximately one-third of the energy consumed as thermal losses and the worldwide $\sim 9400 \mathrm{TWh} / \mathrm{y}$ of mostly low-temperature rejected heat can be recaptured for useful work production. ${ }^{6}$ Closed systems that hybridize energy production technologies with thermal separation methods can access this industrial waste heat, and also low-temperature geothermal energy, using engineered salinity gradients. ${ }^{2}$ Useful work is generated from the controlled mixing of synthetic hypersaline solutions in an energy production stage, while a solution regeneration stage thermally separates the mixture to reconstitute the salinity gradient, essentially converting thermal energy to electricity.

Several approaches have been proposed to harness salinity energy, including pressure retarded osmosis (PRO), ${ }^{7,8}$ reverse electrodialysis (RED), ${ }^{9,10}$ capacitive mixing, ${ }^{11,12}$ osmotically induced nanofluidic electric currents, ${ }^{13}$ and hydrogels. ${ }^{14}$ Among the technologies, membrane-based PRO and RED have been demonstrated at pilot-scale and are considerably more advanced. ${ }^{8,15}$ PRO utilizes the osmotic pressure difference to drive water permeation across a salt-rejecting semipermeable

Received: June 17, 2014

Revised: August 20, 2014

Accepted: August 26, 2014

Published: August 26, 2014 
membrane into a more concentrated "draw" solution. The expanding volume of the draw solution is depressurized through a hydroturbine to produce useful work. RED, on the other hand, is driven by the Nernst potential, another manifestation of the chemical potential difference. The technology employs ion exchange membrane pairs to selectively allow counterion permeation and the net ion flux is converted to an electric current for power generation.

PRO and RED have fundamentally different working principles as well as operating constraints and, thus, are anticipated to have different performance in salinity energy extraction. Furthermore, the permeability and selectivity of the polymeric membranes used in PRO and the ion exchange membranes employed in RED are not at equivalent technological levels. ${ }^{16,17}$ Therefore, it would be instructive to quantitatively analyze the strengths and weaknesses characteristic to the technologies. Evaluation of PRO and RED potential performance with current state-of-the-art membranes can shed light on the viability of harnessing energy from various salinity gradient sources, and reveal vital insights that can inform future development of the technologies. Numerous studies have examined the energy conversion performance of the two processes separately. ${ }^{3,18-22}$ The few that have pitted PRO and RED in direct comparison examined either power density or efficiency, ${ }^{23,24}$ but no study encompassed both metrics.

This study aims to identify the comparative advantages of PRO and RED and examine their practical feasibility for salinity energy extraction. Properties simulating technologically available high performance PRO and RED membranes are employed in the analysis, and trade-off relations governing the membrane parameters are incorporated into the evaluation. The energy efficiency and power density attainable with the simulated membranes are simultaneously assessed for three categories of salinity gradients: natural (seawater-river water), anthropogenic (desalination brine-wastewater effluent), and engineered (synthetic hypersaline solutions). The prospects and limitations intrinsic to PRO and RED are highlighted, and the working principles and primary membrane parameters affecting performance are identified and discussed. The analytical insights of this study can serve to guide membrane and process development for the advancement of PRO and RED energy production from salinity gradients.

\section{ENERGY PRODUCTION FROM SALINITY GRADIENTS}

Both PRO and RED convert the chemical energy stored in salinity gradients to useful work by the controlled mixing of two solutions of different concentrations. However, the two technologies have different working principles, operating considerations, and membrane properties and, thus, are expected to produce distinct power generation performance. In this section, the two technologies are briefly introduced and the governing transport equations are presented. A detailed description of the processes can be found in previous studies. $3,8,10,25$ Here, the fundamental differences between PRO and RED are emphasized, and the different salinity gradients used in this analysis are discussed.

Pressure Retarded Osmosis. Figure 1A shows the schematic of a PRO process, where a semipermeable membrane separates a low concentration (LC) solution and a pressurized high concentration (HC) solution. Because of the difference in salt concentration, an osmotic pressure difference, $\Delta \pi_{\mathrm{m}}$, develops across the membrane that drives water permeation

\section{A Pressure Retarded Osmosis (PRO)}

Semipermeable membrane
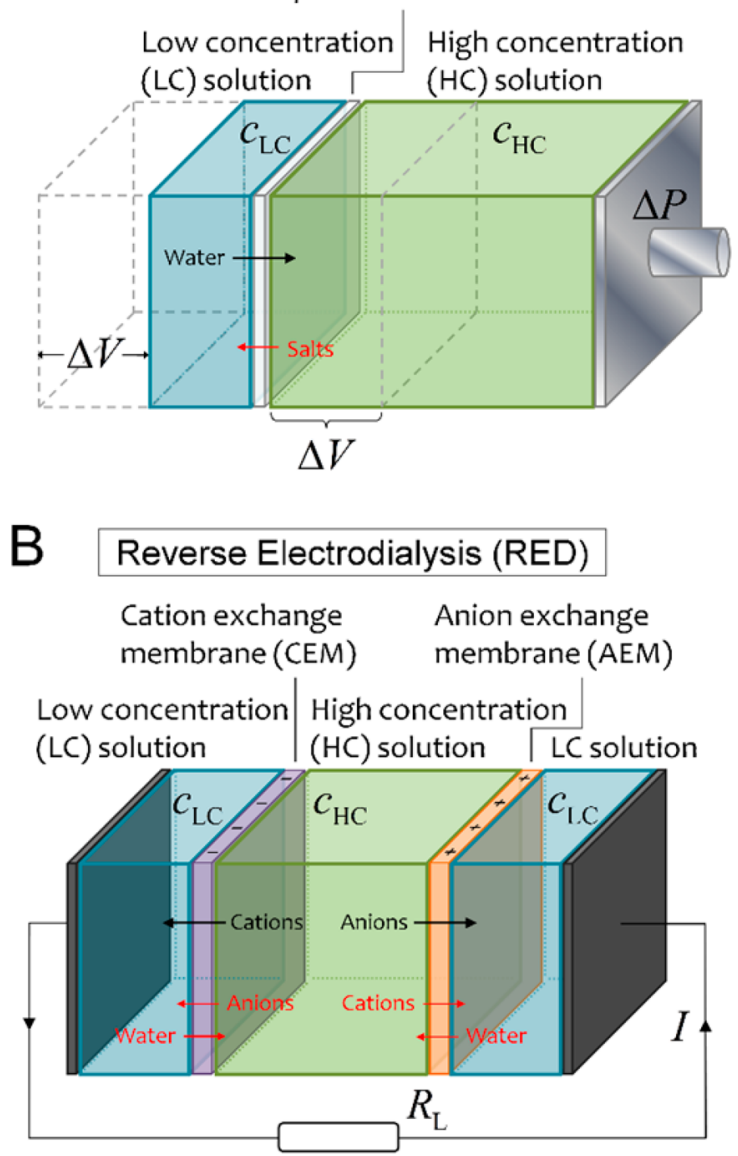

Figure 1. Schematic of (A) pressure retarded osmosis (PRO) and (B) reverse electrodialysis (RED). In PRO, the salinity gradient produces an osmotic driving force for water flux across the semipermeable membrane, and the increasing volume of the pressurized high concentration ( $\mathrm{HC}$ ) solution powers a hydroturbine to produce useful work. Some salt leaks from the HC solution to the low concentration (LC) solution as the membrane is not perfectly selective. In RED, the concentration difference across the ion exchange membranes produces a Nernst potential and the membranes selectively allow the transport of counterions. The ion flux is converted to an electric current, $I$, with a redox couple circulating between the end electrodes and useful work is produced by the external load of resistance $R_{\mathrm{L}}$. Water diffuses to the HC solution, while some co-ions leak across to the LC solution as the membranes are not perfectly selective. Only one RED membrane pair is shown to illustrate the process.

from the LC solution into the more concentrated HC solution. The expanding volume of the pressurized $\mathrm{HC}$ solution powers a hydroturbine to produce useful work. ${ }^{3}$ As the semipermeable membrane is not perfectly selective, some salt diffuses from the saltier side to the more dilute LC solution (Figure 1). The leakage of salts across the membrane represents an uncontrolled mixing that undesirably lowers the extractable energy in PRO.

An actual PRO system would consist of membrane modules in continuous flow operation. ${ }^{8,26}$ Although Figure 1A seemingly depicts a batch process, the model is equivalent to a module operating in cocurrent configuration by assuming ideal plugflow (i.e., the solution concentrations while advancing along the 
axial length of the module correspond to the conditions in the batch process as controlled mixing progresses). The governing equation for water flux in PRO, $J_{w}$, is ${ }^{27}$

$$
\begin{aligned}
J_{\mathrm{w}} & =A\left(\Delta \pi_{\mathrm{m}}-\Delta P\right) \\
& =A\left\{\frac{\pi_{\mathrm{HC}} \exp \left(\frac{-J_{w}}{k}\right)-\pi_{\mathrm{LC}} \exp \left(\frac{J_{\mathrm{w}} S}{D}\right)}{1+\frac{B}{J_{\mathrm{w}}}\left[\exp \left(\frac{J_{\mathrm{w}} S}{D}\right)-\exp \left(\frac{-J_{w}}{k}\right)\right]}-\Delta P\right\}
\end{aligned}
$$

where $\pi_{\mathrm{HC}}$ and $\pi_{\mathrm{LC}}$ are the osmotic pressures of the $\mathrm{HC}$ and LC solutions, respectively, $D$ is the diffusion coefficient of the salt in the LC solution, and $\Delta P$ is the hydraulic pressure applied to the HC solution. The osmotic pressure is determined by the salt concentration, $c$, and can be approximated using the van't Hoff equation: ${ }^{3}$

$$
\pi \approx \nu R_{\mathrm{g}} T c
$$

for relatively dilute solutions $(<1 \mathrm{M} \mathrm{NaCl}) .{ }^{28} \mathrm{Here}, \nu$ is the number of ions each electrolyte molecule dissociates into (i.e., 2 for $\mathrm{NaCl}$ ), $R_{\mathrm{g}}$ is the gas constant, and $T$ is the absolute temperature. A discussion on the validity of the van't Hoff relation can be found in the Supporting Information.

The water permeability coefficient, $A$, the salt permeability coefficient, $B$, and the structural parameter, $S$, are intrinsic properties of the PRO membrane, whereas $k$ is the mass transfer coefficient of the external concentration polarization (ECP) boundary layer at the membrane-HC solution interface. Equation 1 fully accounts for all three performance limiting phenomena in PRO: ECP, internal concentration polarization (ICP), and reverse salt flux. The detailed derivation of the water flux equation and discussion of the performance limiting effects can be found in our previous studies. ${ }^{16,27}$ Inspection of eqs 1 and 2 reveals that PRO water flux and, hence, the rate of controlled mixing is dependent on the membrane properties, $A, B$, and $S$, the hydrodynamic conditions, $k$, and the solution concentrations, $c$.

Reverse Electrodialysis. A schematic of one RED cell is shown in Figure 1B. The HC solution is sandwiched between a pair of cation and anion exchange membranes (CEM and AEM, respectively), which are, in turn, bordered by LC solutions. The charged ion exchange membranes (IEMs) exclude co-ions by Donnan's principle but selectively allow the passage of counterions. The ion concentration difference across the membranes produces a Nernst potential and an ionic current is generated from the directional permeation of ions from the HC solution to the LC solution (Figure 1B). The net ion flow is converted to an electric current, $I$, at the end electrodes with a reversible redox couple and useful work is produced by the external load of resistance, $R_{\mathrm{L}}{ }^{29}$ The IEMs are not perfectly selective and some co-ions undesirably leak across, along with the permeation of water down the osmotic gradient. Akin to PRO, the transport of co-ions and water signifies uncontrolled mixing that subtracts from useful work production. ${ }^{25}$ However, unlike PRO, which converts salinity (chemical) energy to electrical energy via mechanical intermediary, RED directly converts salinity energy to electricity.

An actual RED stack consists of repeating cells arranged in series, ${ }^{25,30}$ but this analysis focuses on a one-cell RED system. By appropriate normalization of the parameters, this apparent inconsistency in system scale is reconciled and the results obtained here are, thus, also applicable for RED stacks. ${ }^{25}$ The current density, $i$, is defined as the current per unit effective membrane area, $A_{\mathrm{m}}$, and can be described by Ohm's law: ${ }^{25}$

$$
i \equiv \frac{I}{A_{\mathrm{m}}}=\frac{\xi_{\mathrm{emf}}-\xi_{\mathrm{L}}}{\mathrm{ASR}_{\text {cell }}}
$$

where $\xi_{\text {emf }}$ is the electromotive force of the RED cell, $\xi_{\mathrm{L}}\left(=I R_{\mathrm{L}}\right)$ is the potential difference across the external load resistor, and $\mathrm{ASR}_{\text {cell }}$ is the area specific resistance of the cell. Adequate mixing at the boundary layer of the membrane-solution interface is assumed such that concentration polarization effects can be neglected. ${ }^{31}$ It is informative to note the symmetry between the PRO water flux equation (eq 1) and the RED current density equation (eq 3 ): $J_{\mathrm{w}}, A, \Delta \pi_{\mathrm{m}}$, and $\Delta P$ are analogous to $i, \mathrm{ASR}_{\text {cell }}{ }^{-1}, \xi_{\text {emf }}$ and $\xi_{\mathrm{L}}$, respectively. Again, by assuming ideal plug flow, the batch process depicted in Figure 1B can similarly represent a continuous flow RED stack with cocurrent circulation of the solutions. ${ }^{25}$

The electromotive force of the RED cell, $\xi_{\mathrm{emf}}$, is the Nernst potential across the IEMs in Figure 1B. For the relatively dilute $\mathrm{NaCl}$ solutions assumed earlier in the PRO description: ${ }^{25}$

$$
\xi_{\mathrm{emf}} \approx \frac{2 \alpha R_{\mathrm{g}} T}{z F} \ln \left(\frac{c_{\mathrm{HC}}}{c_{\mathrm{LC}}}\right)
$$

where $z$ is the ion valence (i.e., 1 for $\mathrm{NaCl}$ ), $F$ is the Faraday constant, and the factor of 2 accounts for the CEM and AEM. The IEM permselectivity, $\alpha$, describes the ability of the membranes to selectively exclude co-ions while allowing the permeation of counterions ( $\alpha=1$ indicates perfect selectivity). The validity of eq 4 over the concentration range investigated is discussed in the Supporting Information.

The RED cell resistance is the series sum of the four stack elements, namely AEM, CEM, the LC solution compartment, and the HC solution compartment. The membrane conductivity is assumed to be constant, whereas the resistance of the solution compartment is taken to be inversely proportional to the solution molar salt concentration. ${ }^{25}$ Hence, the RED current density at any point during the controlled mixing process is determined by the membrane parameters (i.e., conductivity and permselectivity), the stack design (i.e., intermembrane distance), and also the solution properties (i.e., concentrations and resistivity).

Natural, Anthropogenic, and Engineered Salinity Gradients. To examine PRO and RED performance over a range of concentration differences, $\mathrm{HC}-\mathrm{LC}$ solutions pairings of $0.6 \mathrm{M}-1.5 \mathrm{mM} \mathrm{NaCl}$ (seawater-river water), $1.2 \mathrm{M}-10$ $\mathrm{mM} \mathrm{NaCl}$ (desalination brine-wastewater), and 4.0 M-17 $\mathrm{mM} \mathrm{NaCl}$ (hypersaline solution-synthetic brackish water) were employed in this study to simulate natural, anthropogenic, and engineered salinity gradients, respectively. Equal volumes of the HC and LC solutions were used. To simplify the PRO and RED analyses, molar concentration, $c$, was used in all calculations instead of mole fraction, and activity coefficients were assumed to be unity. Accuracy in the Nernst potential is only marginally sacrificed with this approximation $(<7 \%$ difference), ${ }^{32}$ whereas deviation of the initial osmotic pressure difference is more noticeable at high concentrations (approximation underpredicts $\Delta \pi$ by up to $20 \%),{ }^{32}$ but is still within tolerable threshold and does not significantly affect the general comparison between PRO and RED. The differences in driving force for PRO and RED calculated using activities and molar concentrations are analyzed and discussed in the Supporting Information. 


\section{SIGNIFICANT PRO AND RED MEMBRANE PROPERTIES}

Membranes are at the heart of the controlled mixing processes and, therefore, membrane properties and the design of the modules and stacks are expected to have strong bearing on PRO and RED performance. Salient parameters of the saltselective membranes and ion exchange membranes analyzed in this study are highlighted here, and significant factors of the module and stack are briefly discussed. The parameters are selected to simulate state-of-the-art innovations and, hence, the results presented in this analysis roughly circumscribe the potential PRO and RED performance achievable with current technology.

Structural Parameter and Mass Transport Coefficient of PRO Membranes. Internal concentration polarization (ICP) is an important performance limiting phenomenon that lowers power density in PRO by detrimentally elevating the membrane active-support layer interfacial salt concentration. ${ }^{16,27,33}$ The extent of ICP is determined by the structural parameter, $S$, of the membrane support layer, as reflected in eq 1 by the term $\exp \left(J_{\mathrm{w}} S / D\right)$. At the same time, pressurized PRO operation imposes mechanical strength requirements: the membrane needs to be adequately robust to withstand $\Delta P .^{34,35}$ An $S$ value of $500 \mu \mathrm{m}$ was selected to simulate commercially manufactured membranes with polyester woven mesh embedded in a highly porous and nontortuous polysulfone support layer. ${ }^{35}$ These membranes were experimentally demonstrated to possess mechanical sturdiness and substantially suppress ICP. Additionally, external concentration polarization (ECP) at the solution-active layer interface was found to be a dominant performance limiting effect at high water fluxes. ${ }^{16}$ Mass transfer coefficient, $k$, of the ECP boundary layer is taken to be $27.5 \mu \mathrm{m} / \mathrm{s}$ to model PRO membrane modules with high performance channel and spacer design. ${ }^{35}$ The parameters employed in this analysis are summarized in Table 1.

PRO Membrane Selectivity-Permeability Trade-off. An inspection of eq 1 reveals that water flux can be enhanced by using a membrane with large water permeability, $A$, and small salt permeability, $B$. A high $A$ allows greater water permeation whereas a low $B$ curbs the leakage of salt from the HC solution. The unfavorable buildup of leaked salt in the membrane support layer diminishes the osmotic driving force. $^{16,27}$ Furthermore, salt permeation across the PRO membrane constitutes uncontrolled mixing that detrimentally deducts from useful work production. However, the goals of raising $A$ and minimizing $B$ cannot be simultaneously achieved due to the permeability-selectivity trade-off governing saltrejecting polymeric membranes based on the solution diffusion mechanism of transport. ${ }^{36}$ For example, our recent study on polyamide thin-film composite membranes indicates that doubling $A$ would concomitantly increase $B$ by 8 -fold. ${ }^{16}$

Polyamide membranes are the state-of-the-art for reverse osmosis desalination ${ }^{37,38}$ and also extensively researched for PRO applications. ${ }^{35,39-41}$ To simulate technically attainable polyamide membranes of different permeability-selectivity, two pairs of parameters based on the trade-off correlation equation developed in our previous work ${ }^{16}$ were chosen for this analysis (Table 1): $A=1.5 \mathrm{~L} \mathrm{~m}^{-2} \mathrm{~h}^{-1} \mathrm{bar}^{-1}, B=1.25 \times 10^{-8}$ $\mathrm{m} / \mathrm{s}$ and $A=3.0 \mathrm{~L} \mathrm{~m}^{-2} \mathrm{~h}^{-1} \mathrm{bar}^{-1}, B=10 \times 10^{-8} \mathrm{~m} / \mathrm{s}$ (designated I and II, respectively). Recent studies suggest that membrane properties can be altered under pressurized
Table 1. Properties of Current High Performance Membranes (Thin-Film Composite Polyamide Membranes for PRO and Ion Exchange Membranes for RED) Analyzed in This Study

\begin{tabular}{|c|c|c|}
\hline & Selectivity I & Selectivity II \\
\hline \multicolumn{3}{|c|}{ PRO membranes } \\
\hline $\begin{array}{l}{ }^{a} \text { water permeability, } A \\
\left(\mathrm{~L} \mathrm{~m}^{-2} \mathrm{~h}^{-1} \mathrm{bar}^{-1}\right)\end{array}$ & 1.5 & 3.0 \\
\hline $\begin{array}{l}a_{\text {salt }}(\mathrm{NaCl}) \text { permeability, } B \\
\left(10^{-8} \mathrm{~m} / \mathrm{s}\right)\end{array}$ & 1.25 & 10 \\
\hline${ }^{b}$ structural parameter, $S(\mu \mathrm{m})$ & 500 & 500 \\
\hline \multicolumn{3}{|c|}{ RED membranes } \\
\hline${ }^{c}$ area specific resistance, ASR $\left(\Omega \mathrm{cm}^{2}\right)$ & 3.0 & 1.5 \\
\hline$c, d$ permselectivity, $\alpha(-)$ & $0.95 / 0.90 / 0.80$ & $0.90 / 0.80 / 0.60$ \\
\hline${ }^{d}$ molar water permeation ratio, $\theta(-)$ & $3.5 / 11 / 26$ & $19 / 44 / 94$ \\
\hline
\end{tabular}

${ }^{a}$ Water and salt permeability of the PRO membranes are based on the permeability-selectivity trade-off relationship of polyamide thin-film composite membranes. ${ }^{16} b_{\text {The structural parameter is selected to }}$ simulate a membrane with woven fabric support capable of withstanding high hydraulic pressures. ${ }^{35}$ Area specific resistance and permselectivity values are chosen to simulate technologically available high performance ion exchange membranes constrained by conductivity-permselectivity trade-off. ${ }^{43}{ }^{d}$ Permselectivity of the IEMs is assumed to deleteriously decrease with increasing salt concentration $(0.6 / 1.2 / 4.0 \mathrm{M} \mathrm{NaCl})$ of the surrounding solutions, while the molar water permeation ratio detrimentally rises. ${ }^{49}$

conditions. $^{35,42}$ For this analysis, the spacer design of the PRO module was assumed to adequately support the membrane such that $A, B$, and $S$ are constant and independent of $\Delta P$.

RED Membrane Ionic Permselectivity-Conductivity Trade-off. Recent studies indicate an analogous trade-off exists for ion exchange membranes that relates permselectivity, $\alpha$, and ionic conductivity. ${ }^{43,44}$ Specifically, an increase in $\alpha$ is generally accompanied by an undesired rise in the area specific resistance, ASR, of AEMs. The observed trend was attributed to fixed charge density and water content of the polymeric membranes ${ }^{43,44}$ and, therefore, CEMs should also exhibit similar behavior. Our recent study showed that membrane resistance is a crucial performance factor and a low ASR is advantageous for RED. ${ }^{25}$ On the other hand, an $\alpha$ close to unity is desired to maximize the Nernst potential across the RED cell, as in eqs 3 and 4, and also restrain the leakage of co-ions that represents uncontrolled mixing.

Additionally, IEMs are hydrated polymeric thin-films and are, thus, pervious to water. ${ }^{17}$ Water permeation down the osmotic gradient, into the $\mathrm{HC}$ solution, constitutes uncontrolled mixing that further subtracts from useful work production. Water leakage across the membranes can be described by $\theta$, the mole ratio of water to salt (both counterions and co-ions) permeation. ${ }^{25}$ In the absence of a rigorous empirical equation linking $\alpha$, ASR, and $\theta$, two sets of conductivity-permselectivity were identified from literature to reflect technologically feasible IEMs (Table 1): ${ }^{17,25,45,46}$ ASR $=3.0 \Omega \mathrm{cm}^{2}, \alpha=0.95, \theta=3.5$ and ASR $=1.5 \Omega \mathrm{cm}^{2}, \alpha=0.90, \theta=19$ (likewise designated I and II, respectively). The cation and anion exchange membranes are assumed to have the same conductivity and symmetrically identical selectivity. Similar to the assumptions for PRO membranes, the properties are taken to be constant during RED.

Effect of Solution Concentration and Intermembrane Distance. Ion exchange membranes utilize the Donnan 
exclusion principle to allow the selective permeation of counterions while retaining co-ions. The fixed charges of IEM depress the co-ion concentration within the membrane because of charge balance, thus effectively excluding their transport across the IEM. As the ratio of counter to co-ions in the membrane matrix is approximately proportional to the square of the ratio of fixed charge density to surrounding solution concentration, the exclusion effect of the fixed charges is diminished when the solution concentration is high. ${ }^{47,48}$ Hence, IEM selectivity is reduced with increasing concentration of the surrounding solution, as experimentally demonstrated in a recent study where $\alpha$ of commercial CEM and AEM decreased from $>0.95$ at $\mathcal{c}_{\mathrm{HC}}$ of $\sim 0.5 \mathrm{M} \mathrm{NaCl}$, to $\sim 0.80$ at elevated concentrations of $4 \mathrm{M} \mathrm{NaCl}$. ${ }^{49}$ To simulate the deteriorating permselectivity at the high salt concentrations of anthropogenic and engineered salinity gradients, $\alpha$ of RED-I is reduced to 0.90 and 0.80 , respectively, $\alpha$ of RED-II is lowered to 0.80 and 0.60 , and $\theta$ is revised accordingly to reflect the greater water permeation (Table 1).

Apart from the IEMs, the HC and LC solutions contribute to the area specific resistance of the RED cell. As the conductivity of the solution compartment is inversely proportional to the channel height, a small intermembrane distance is advantageous to minimize $\mathrm{ASR}_{\text {cell }}$ and, thus, enhance power density. On the other hand, a narrow channel height exacerbates parasitic pressure drop along the channels and increases the pumping energy cost. ${ }^{25,50}$ To model a well-designed RED stack, an intermembrane distance of $150 \mu \mathrm{m}$ that balances the benefits of lower cell resistance with the negative effects of pressure drop is selected. $^{25}$ Additionally, adequate mixing at the solutionmembrane interface is assumed such that the effects of concentration polarization are negligible. ${ }^{25,50}$

\section{SALINITY ENERGY PRODUCTION PERFORMANCE PARAMETERS}

Membrane power density, PD, and energy extraction efficiency, $\eta$, are two primary performance parameters affecting the costeffectiveness of power generation from salinity gradients using membrane-based technologies., ${ }^{3,8,17,25} \mathrm{PD}$ is defined as the power produced per unit membrane area and is a measure of how quickly the membranes convert salinity energy to useful work, whereas $\eta$ is the percent of Gibbs free energy of mixing converted to useful work and, thus, quantifies how much of the available energy is utilized by the process. In this section, the significance of the performance metrics is outlined and the practical operating conditions for PRO and RED are briefly discussed.

Membrane Power Density. Membrane is a key capital cost component for both PRO and RED, ${ }^{51,52}$ and a high PD is desirable to reduce the membrane area required for generating a certain power. Membrane power density for PRO and RED, respectively, are

$$
\begin{aligned}
& \mathrm{PD}_{\mathrm{PRO}}=J_{\mathrm{w}} \Delta P \\
& \mathrm{PD}_{\mathrm{RED}}=\frac{1}{2} i \xi_{\mathrm{L}}
\end{aligned}
$$

Note that RED innately requires two membranes, CEM and AEM, whereas PRO requires just one salt-rejecting membrane (Figure 1). This difference is reflected in eq 6 by the factor of $1 / 2$. To simulate practical operation, this analysis considers the hydraulic pressure applied to the HC solution of the PRO membrane module and the external load of the RED circuit to be both constant throughout the controlled mixing., ${ }^{3,25}$ As the processes progress, which is equivalent to advancement down the PRO module or RED stack, the HC solution is diluted and $c_{\mathrm{LC}}$ increases, consequently changing $J_{\mathrm{w}}, i$, and $\xi_{\mathrm{L}}$. Therefore, the immediate power density, described by eqs 5 and 6 , varies accordingly. To evaluate the overall PRO and RED performance, $\mathrm{PD}$ is averaged over the entire membrane module or stack to yield the net power density.

Energy Extraction Efficiency. The Gibbs free energy of mixing, $\Delta G_{\text {mix }}$ for strong electrolyte solutions of relatively dilute concentrations ${ }^{3}$

$$
-\frac{\Delta G_{\mathrm{mix}}}{\nu R_{\mathrm{g}} T} \approx c_{\mathrm{M}}^{\mathrm{f}} \ln c_{\mathrm{M}}^{\mathrm{f}}-\phi_{\mathrm{LC}} \mathcal{c}_{\mathrm{LC}}^{0} \ln c_{\mathrm{LC}}^{0}-\phi_{\mathrm{HC}} \mathcal{c}_{\mathrm{HC}}^{0} \ln c_{\mathrm{HC}}^{0}
$$

gives the theoretical maximum energy, per unit total solution volume, from the complete mixing of the $\mathrm{HC}$ and $\mathrm{LC}$ solutions. The subscript $M$ denotes the resultant mixture, the superscripts 0 and $f$ indicate initial and final states, and $\phi$ is the volume fraction $\left(\phi_{\mathrm{LC}}=\phi_{\mathrm{HC}}=0.5\right.$ for the equivolume analyses in this study). To fully access $\Delta G_{\text {mix }}$ for useful work would require a hypothetical reversible thermodynamic process. Practical unit operations are, however, irreversible in nature and inevitably produce entropy that reduces the efficiency, $\eta$. Due to fundamentally different working principles and dissimilar mode of practical operation, PRO and RED have inherent efficiencies that are characteristic of the processes. When comparing PRO and RED, it is, hence, instructive to examine the thermodynamic efficiency intrinsic to the technologies.

Practical Operation of PRO and RED. Detailed PRO and RED operating parameters and behavior are discussed in the Supporting Information, whereas key parameters and performance objectives employed in this study are briefly presented here. Our recent work indicates that because the driving force is gradually diminished as controlled mixing progresses, judicious early discontinuation of PRO and RED can yield significantly enhanced PD with only marginal forfeit in $\eta^{25}$ To model practical operation, triggers for early process termination were rationally selected (after $70 \%$ of the eventual ion permeation in $\mathrm{RED}^{25}$ and, based on similar criteria, after $90 \%$ of the eventual water permeation in PRO). The comparisons presented here focus on RED performance when PD is maximized and PRO performance when $\eta$ is maximized (the asymmetric performance objectives are further discussed in the Supporting Information). All power densities and efficiencies were analyzed numerically with temperature $T=298 \mathrm{~K}$. Additionally, only cocurrent flow is considered for the simulated PRO module and RED stack unit operation; greater mixing can be achieved with counter- or cross-current configuration, thus accessing more $\Delta G_{\text {mix }}$ for energy production. ${ }^{19,53}$ Methodology for RED module analysis is detailed in our recent work ${ }^{25}$ and is adapted here, together with the energy analysis approach employed in our earlier study, ${ }^{3}$ for evaluating both PRO and RED.

\section{ENERGY EXTRACTION EFFICIENCY}

Efficiency, Entropy Production, and Unutilized Energy. For equal volumes of $\mathrm{HC}$ and LC solution, the Gibbs free energy of mixing for natural, anthropogenic, and engineered salinity gradients are $0.56,1.08$, and $3.68 \mathrm{kWh}$ per $\mathrm{m}^{3}$ of LC solution volume, respectively, as determined using eq 7 with $\phi_{\mathrm{LC}}=0.5$. Alternatively, the energy can be expressed per cubic meter of the total (mixed) solution, ${ }^{10,19}$ in which case the 
corresponding specific $\Delta G_{\text {mix }}$ are half of the above values as $\phi_{\mathrm{LC}}$ $=0.5$. In irreversible unit operation, $\Delta G_{\text {mix }}$ is fractionated into useful work generated, entropy produced due to friction/ resistance, entropy produced from uncontrolled mixing, and energy not utilized. ${ }^{3,25}$ The energy allotment is depicted in Figure 2 for natural salinity gradient, where the driving force,

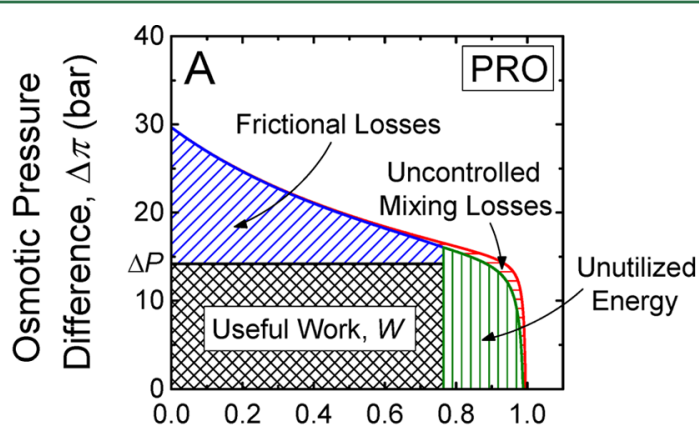

Fraction of LC Solution Permeated, $\triangle V I V_{\mathrm{LC}}^{0}$

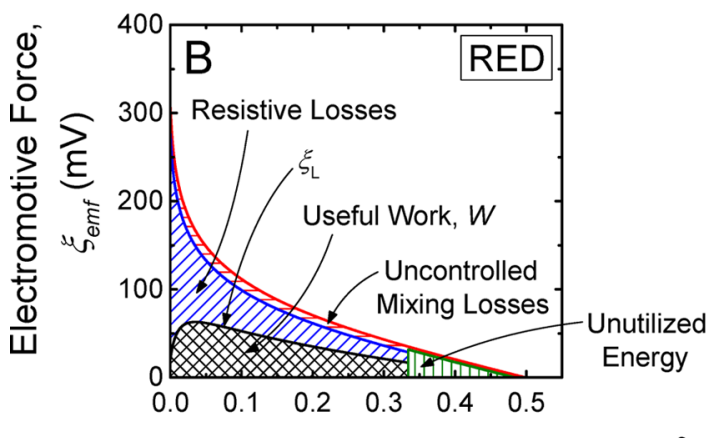

Fraction of HC Salt Permeated, $\Delta n_{\mathrm{s}} / n_{\mathrm{s}, \mathrm{HC}}^{0}$

Figure 2. Representative plots of useful work, $W$ (black patterned areas), frictional/resistive losses (blue patterned areas), uncontrolled mixing losses (red patterned areas), and unutilized energy (green patterned areas) for A) PRO and B) RED. The vertical axes are the driving force for water and ion flux (osmotic pressure difference, $\Delta \pi$, and electromotive force, $\xi_{\text {emf }}$ for PRO and RED, respectively), whereas the horizontal axes denote progress of the energy production process (fraction of water permeated from the low concentration (LC) solution, $\Delta V / V_{\mathrm{LC}}^{0}$, and fraction of salt permeated from the high concentration (HC) solution, $\Delta n_{\mathrm{s}} / n_{\mathrm{s}, \mathrm{HC}}^{0}$, respectively). For both processes, the $\mathrm{HC}$ solution is $0.6 \mathrm{M} \mathrm{NaCl}$ and the LC solution is $1.5 \mathrm{mM} \mathrm{NaCl}$ to simulate seawater-river water salinity gradient. Membrane properties are presented in the column "Selectivity I" of Table 1. To maximize $\eta, \Delta P$ in PRO is $14.2 \mathrm{bar}$ (about half of initial $\Delta \pi)$ whereas $R_{\mathrm{L}} A_{\mathrm{m}}=9.15 \Omega \mathrm{cm}^{2}$ in RED to maximize PD.

$\Delta \pi$ and $\xi_{\text {emf }}$, is plotted as a function of fraction of water permeated for PRO and salt permeated for RED. Membranes possessing properties listed in "Selectivity I" column of Table 1 were used in the analysis. To maximize $\eta, \Delta P$ in PRO is 14.2 bar whereas $R_{\mathrm{L}} A_{\mathrm{m}}=9.15 \Omega \mathrm{cm}^{2}$ in RED to maximize PD. The methodology to determine the utilization and distribution of the available energy can be found in our recent publications. ${ }^{3,25}$

The black patterned region in Figure $2 \mathrm{~A}$ denotes the useful work produced in constant-pressure PRO when energy efficiency is maximized, and is obtained by multiplying the applied hydraulic pressure, $\Delta P$, and the volume of water permeated in the PRO module, i.e., area under $\Delta P-\Delta V$. Because practical PRO is not a reversible thermodynamic process, entropy is inevitably produced. First, water permeating across the semipermeable membrane is impeded by water-membrane frictional forces. Energy is expended to overcome the hydraulic resistance and achieve a nonzero water flux. ${ }^{3}$ Entropy production due to frictional losses is indicated in Figure 2A as the blue patterned region. Second, the semipermeable membranes are not perfectly selective and some salts leak across. Thus, energy is lost from the uncontrolled mixing of the salinity gradient caused by the undesired salt flux. The red patterned region in Figure 2A marks the entropy production because of uncontrolled mixing. Both frictional losses and uncontrolled mixing render PRO thermodynamically irreversible. Lastly, the green patterned area denotes the portion of $\Delta G_{\text {mix }}$ that is unutilized. As PRO progresses, the net driving force gradually diminishes and water flux is eventually terminated when $\Delta \pi-\Delta P=0$. The remaining energy still embedded in the unmixed solutions is, hence, not accessed. This characteristic is a practical limitation of constant-pressure PRO. ${ }^{3}$ Additionally, the portion of unutilized energy is slightly augmented by the early discontinuation of PRO (discussed in previous section). Aggregating the four regions under the osmotic driving force curve yields $\Delta G_{\text {mix }}$ (Figure 2A).

The mixing energy can similarly be partitioned for RED when power density is maximized, as represented by division of the area under $\xi_{\text {emf }}$ in Figure 2B. Integrating the potential difference of the external load, $\xi_{\mathrm{L}}$, across the moles of salt permeated as counterions (equivalently, the charge transported) gives the useful work produced (black patterned region). ${ }^{25}$ Note that unlike constant-pressure $\mathrm{PRO}, \xi_{\mathrm{L}}$ varies over the course of RED due to the changing solution concentrations and $\xi_{\text {emf }}$. Internal resistance of the RED stack, caused by the IEMs and the electrolyte solutions, dissipates energy by impeding the permeations of ions. Entropy production due to resistive energy loss in RED is indicated as the blue patterned area in Figure $2 \mathrm{~B}$, and is analogous to frictional energy loss in PRO. Similar to PRO, uncontrolled mixing in RED with real membranes also produces entropy (red patterned region): water and co-ions leaks across the IEMs as the membranes are not perfectly selective. Whereas constant $\triangle P$ in PRO restricts the complete mixing of the $\mathrm{HC}$ and LC solutions, RED with constant $R_{\mathrm{L}}$ can proceed to concentration equilibrium (i.e, $c_{\mathrm{HC}}=c_{\mathrm{LC}}$ ). Therefore, a greater portion of $\Delta G_{\text {mix }}$ can be accessed by RED. The green patterned region in Figure $2 \mathrm{~B}$ denotes the unutilized energy due to premature discontinuation of the process for the reason discussed in the previous section.

Higher Efficiency is Attainable in PRO than RED. The useful work, frictional/resistive losses, uncontrolled mixing losses, and unutilized energy, as illustrated in Figure 2, can be expressed as a percent of the total available energy (by taking the ratio of the black, blue, red, and green patterned areas, respectively, to the total area under the curve). Gray columns in Figure 3 show PRO and RED efficiency, $\eta$ (defined as the percent of $\Delta G_{\mathrm{mix}}$ converted to useful work), for natural, anthropogenic, and engineered salinity gradients. Membranes with Selectivity I (Table 1) were used in the analysis. With the practical operating conditions described earlier, PRO exhibits greater energy extraction efficiency (53.9, 53.9, and 56.1\%) than RED (37.8, 33.1, and 18.1\%) for all three salinity gradients. Note that RED can potentially attain higher $\eta$, but at the expense of drastically reduced power density, thus rendering the overall process impractical (further discussion can be found in the Supporting Information). Frictional/ resistive energy losses, uncontrolled mixing losses, and 


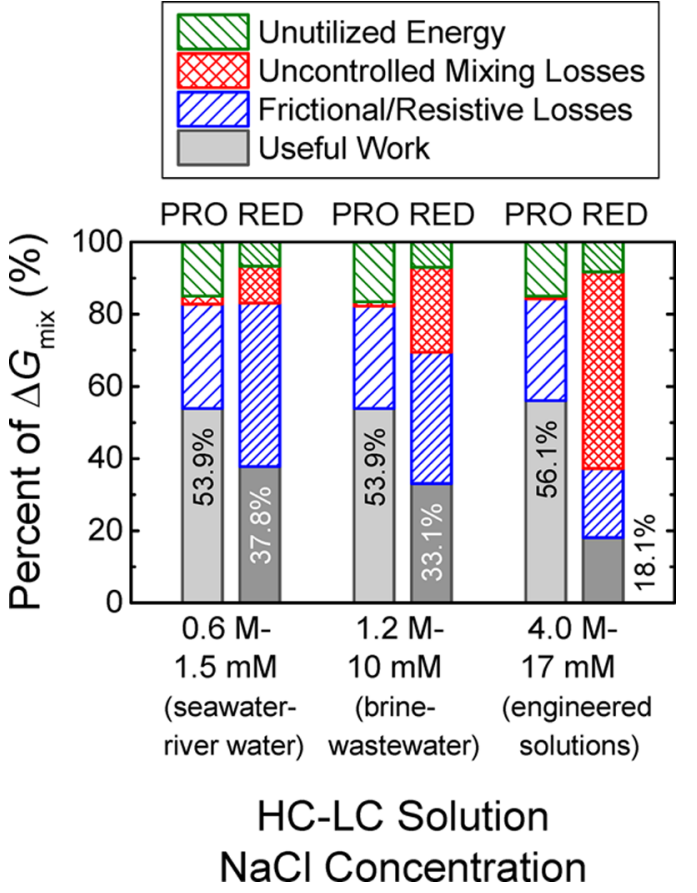

Figure 3. Efficiency of work extraction (gray columns), percent of energy expended to drive water or counterion flux (blue patterned columns), percent of energy lost to uncontrolled mixing (red patterned columns), and portion of unutilized energy (green patterned columns) for PRO and RED. The HC-LC solution concentrations are $0.6 \mathrm{M}-1.5 \mathrm{mM}, 1.2 \mathrm{M}-10 \mathrm{mM}$, and $4.0 \mathrm{M}-17 \mathrm{mM} \mathrm{NaCl}$ to simulate seawater-river water, seawater desalination plant brinewastewater, and engineered solutions, respectively. The PRO and RED membrane properties are presented in the column "Selectivity I" of Table 1.

unutilized energy are also presented (blue, red, and green patterned columns, respectively).

Because of the constant pressure limitation, PRO has inherently larger fractions of unutilized energy (green patterned columns) than RED. However, less entropy is produced in PRO than RED (blue and red patterned columns combined). Numerical values of the percent of entropy produced and energy not utilized are presented in Table S1 of the Supporting Information. The relatively superior selectivity of salt-rejecting membranes to the co-ion and water retention capabilities of IEMs enables PRO to better suppress uncontrolled mixing (red patterned columns). This RED disadvantage is especially pronounced at larger salinity gradients (i.e., anthropogenic and engineered), where the high concentration of the $\mathrm{HC}$ solution significantly suppresses the Donnan exclusion capacity of the ion exchange membranes and detrimentally diminishes the ability of RED to convert salinity energy to useful work. ${ }^{49}$ Even when IEM selectivity is not compromised in the natural salinity scenario, a larger portion of $\Delta G_{\text {mix }}$ is lost to entropy production due to internal stack resistance in RED than watermembrane friction in PRO (blue patterned columns). For the range of salinity gradients, the analysis indicates that PRO offers a greater energy extraction efficiency advantage over RED.

Selective Membranes Yield Greater Efficiencies. PRO and RED efficiencies with different membrane transport parameters (Selectivity I and II of Table 1) are presented in Figure 4 (unshaded and shaded symbols, respectively, and right vertical axis) for natural, anthropogenic, and engineered salinity gradients. Both energy extraction technologies yield greater
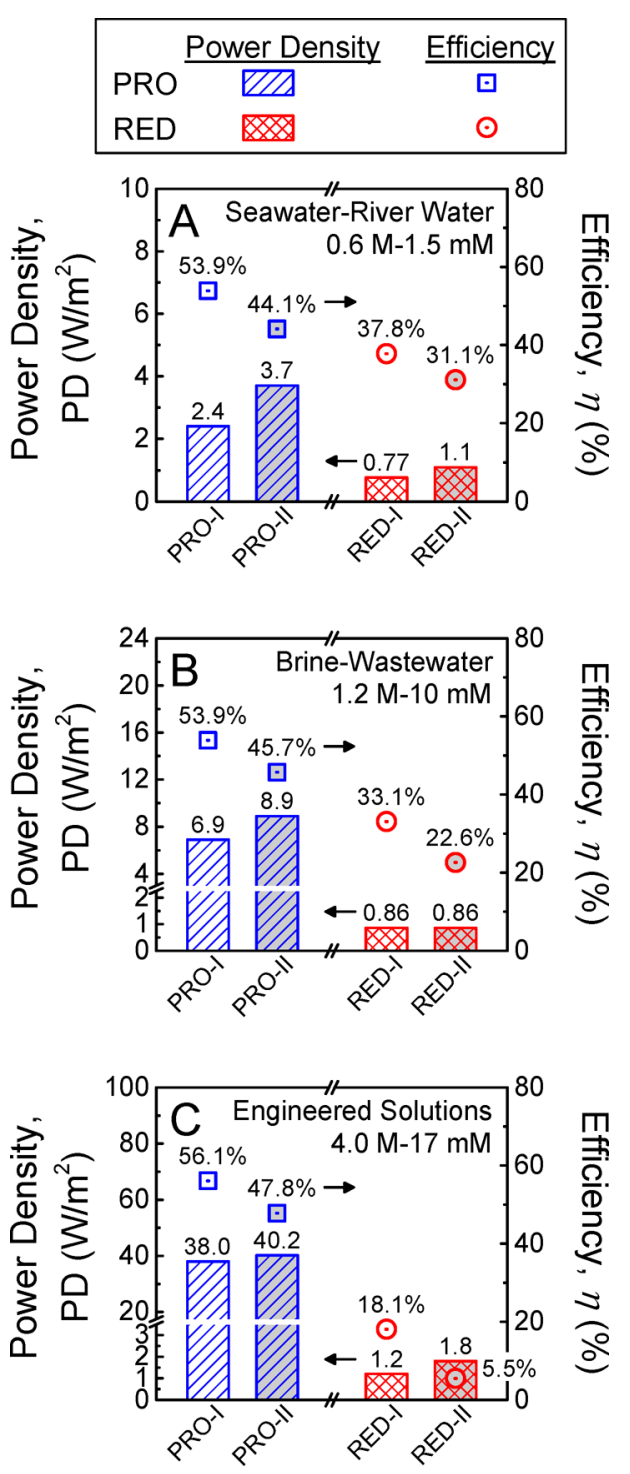

Figure 4. Membrane power density (columns, left vertical axis) and efficiency of work extraction (symbols, right vertical axis) for PRO and RED (blue and red data representations, respectively) at $0.6,1.2$, and 4.0 M NaCl high concentration (HC) solution concentrations. Two sets of membranes with imperfect selectivity are examined: membrane-I has moderate selectivity imperfections (unshaded columns and symbols), whereas membrane-II has more severe imperfection in selectivity (shaded columns and symbols). Details of the PRO and RED membrane properties can be found in Table 1.

efficiencies with more selective membranes (PRO-I and RED-I) for all three salinity gradient scenarios. This result is unsurprising, as entropy production due to uncontrolled mixing is lower because less salt and co-ions leak across in PRO and RED, respectively, when membranes of higher selectivity are used. Table S1 of the Supporting Information shows the complete data set for entropy production and energy unutilized, along with the applied hydraulic pressure in PRO and load resistance in RED. The disparity in $\eta$ is most evident in RED with anthropogenic and engineered salinity gradients, as the high solution concentrations significantly suppress Donnan exclusion, thereby causing greater co-ion leakage. As discussed in the following section, despite the seeming drawback in efficiency, employing less selective membranes can be advanta- 
geous for overall productivity because of potentially greater power densities.

\section{MEMBRANE POWER DENSITY}

Higher Power Density Obtained with Less Resistive Membranes. Power density, defined as the membrane areanormalized power generation, is an important factor affecting the cost-effectiveness of PRO and RED. ${ }^{51,52}$ Unshaded and shaded columns of Figure 4A-C (left vertical axis) denote the power densities, PD, for Selectivity I and II membranes, respectively. Compared to the more selective membranes, PRO-II is twice as permeable (Table 1) and is able to attain a higher $\mathrm{PD}$ in the cocurrent operation for natural, anthropogenic, and engineered salinity gradients $(2.4,6.9$, and 38.0 $\mathrm{W} / \mathrm{m}^{2}$ for PRO-I, respectively, and $3.7,8.9$, and $40.2 \mathrm{~W} / \mathrm{m}^{2}$ for PRO-II). However, this PD advantage is achieved at the expense of efficiency: $\eta$ is $53.9-56.1 \%$ for PRO-I but is lower for PRO-II (44.1-47.8\%) due to greater entropy production arising from more salt leakage.

The relative PD enhancement is more marked for smaller salinity gradients. For instance, by using membranes with lower hydraulic resistance, PD is augmented 54\% with seawater-river water but only improved 6\% with engineered hypersaline solutions. Increasing water permeability concomitantly raises salt permeability because of the permeability-selectivity tradeoff governing salt-rejecting membranes. ${ }^{16,27}$ As the detrimental effect of reverse salt flux coupled with internal concentration polarization is more exacerbated at higher water fluxes, PD enhancements, therefore, do not scale with the water permeability increases (eqs 1 and 5). Additionally as PRO progresses, more draw salt is accumulated in the feed solution for high $c_{\mathrm{HC}}$ that deleteriously reduces the effective $\Delta \pi$ across the membrane and further lowers PD. Hence, employing more selective PRO-I membranes for engineered salinity gradients can be overall more productive, as $\eta$ is substantially higher (56.1\%) while PD is only marginally diminished $\left(38.0 \mathrm{~W} / \mathrm{m}^{2}\right)$ compared to $47.8 \%$ and $40.2 \mathrm{~W} / \mathrm{m}^{2}$ of PRO-II (Figure $4 \mathrm{C}$ ).

Less resistive RED membranes exhibit a generally similar trend for the cocurrent stacks: PD of $0.77-1.2 \mathrm{~W} / \mathrm{m}^{2}$ is obtained with Selectivity I, whereas Selectivity II yields higher power densities of $0.86-1.8 \mathrm{~W} / \mathrm{m}^{2}$ (red columns of Figure 4). Ion exchange membranes are also bound by an analogous trade-off, ${ }^{43,44}$ where an increase in membrane conductivity is unavoidably accompanied by a reduction in permselectivity (Table 1). However, the net effect of the permselectivityconductivity trade-off on the resultant PD is less straightforward. A lower resistance enables greater ionic flux across the membranes and, hence, enhances PD (eqs 3 and 6) but the Nernst potential to drive ion permeation is diminished by the associated decline in permselectivity (eq 4). Detrimental lowering of the permselectivity by highly concentrated solutions further compounds to the complexity. The convoluted behavior is illustrated by more conductive RED-II membranes obtaining PD enhancement of $43 \%$ and $50 \%$ for natural and engineered salinity gradients, respectively, but no noticeable benefits for the brine-wastewater system.

PRO Better Utilizes Salinity Gradient to Realize Greater Power Densities. Appreciably greater membrane power densities are attainable in PRO than RED (blue and red columns in Figure 4). For natural, anthropogenic, and engineered salinity gradients, respectively, $\mathrm{PD}_{\mathrm{PRO}}$ is $3.1-3.4$ times, 8-10 times, and 22-32 times of $\mathrm{PD}_{\mathrm{RED}}$, indicating that with the simulated state-of-the-art membrane properties, PRO requires significantly less membrane area to access the salinity energy. The PD difference between the two membrane-based energy production technologies is amplified at larger salinity gradients. Contrary to intuition, the relatively poorer PD performance of RED in high salt concentrations conditions is not solely caused by the diminishing membrane selectivity. Using simulated RED membranes with $\alpha=0.95, \theta=3.5$, and ASR $=3.0 \Omega \mathrm{cm}^{2}$ (i.e., assuming selectivity is not compromised by solution concentration) in engineered salinity gradient yields higher $\eta$ of $37.7 \%$, but conspicuously no noticeable PD improvements $\left(1.2 \mathrm{~W} / \mathrm{m}^{2}\right)$ compared to $18.1 \%$ and $1.2 \mathrm{~W} / \mathrm{m}^{2}$ of RED-I.

The superior power density performance of PRO over RED at larger salinity gradients can be elucidated by examining the driving force for water and ion flux. PRO osmotic pressure difference, $\Delta \pi$, as a function of fraction of LC solution permeated, and RED electromotive force, $\xi_{\text {emf }}$ as a function of salt permeated (as counterions) per $\mathrm{HC}$ solution volume are presented in Figure 5 (inset of Figure 5B shows the initial $\xi_{\text {emf }}$ ). This analysis assumes ideal, perfectly selective membranes (i.e., $B=0$, and $\alpha=1$ and $\theta=0$ ). For such ideal membranes, the
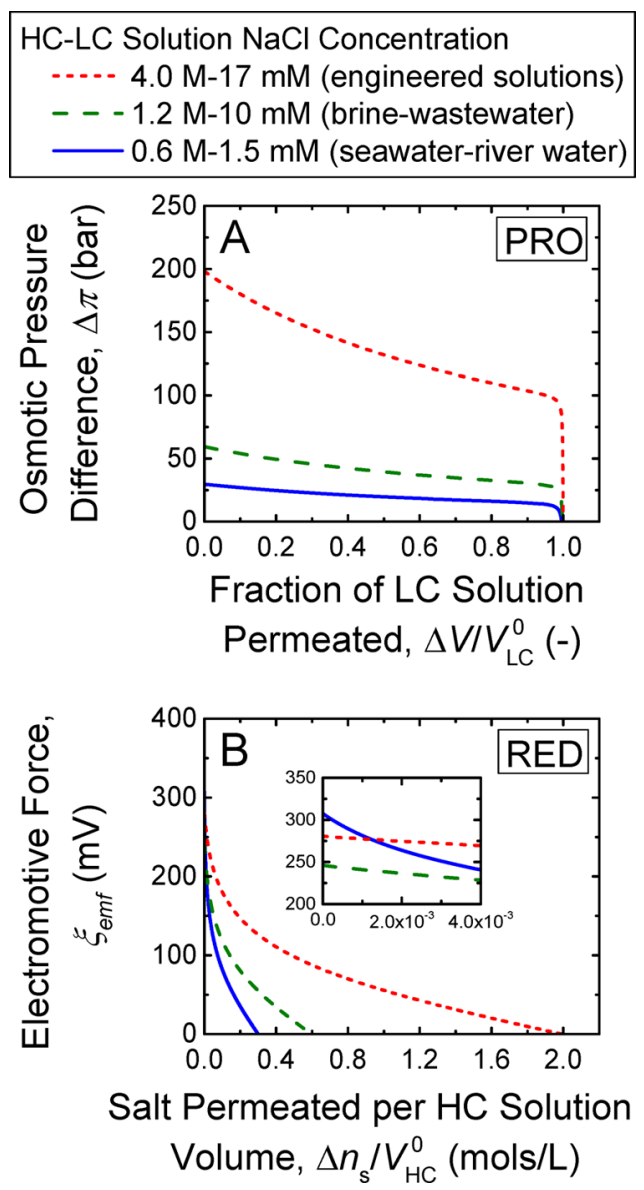

Figure 5. (A) Osmotic pressure difference, $\Delta \pi$ (driving force for water flux in PRO), as a function of the fraction of water permeated from the low concentration (LC) solution, $\Delta V / V_{\mathrm{LC}}^{0}$ (B) Electromotive force, $\xi_{\text {emf }}$ (driving force for ion flux in RED), as a function of the moles of salt permeated per unit volume of the high concentration (HC) solution, $\Delta n_{\mathrm{s}} / V_{\mathrm{HC}}^{0}$. Inset in panel B shows the initial $\xi_{\mathrm{emf}}$ of the RED cell. Blue solid, green dashed, and red dotted lines represent natural, anthropogenic, and engineered salinity gradients, respectively. Perfectly selective PRO and RED membranes are assumed. 
area under the blue solid, green dashed, and red dotted lines is proportional to the theoretical maximum energy $\Delta G_{\text {mix }}(0.56$, 1.08 , and $3.68 \mathrm{kWh}$ per $\mathrm{m}^{3}$ of LC solution volume) for natural, anthropogenic, and engineered salinity gradients, respectively.

The Gibbs free energy of mixing is determined by the solution concentrations (eq 7) and is independent of the energy extraction technologies. More salinity energy is available with larger concentration differences, but PRO and RED access the higher $\Delta G_{\text {mix }}$ in fundamentally different ways. In PRO, the fraction of LC solution that permeates across is practically the same (horizontal axis-intercept of Figure 5A), but $\Delta \pi$ increases proportionally with $c_{\mathrm{HC}}$ (eq 2 and Figure $5 \mathrm{~A}$ ). For example, increasing $c_{\mathrm{HC}}$ by 6.7 times from 0.6 to $4.0 \mathrm{M} \mathrm{NaCl}$ raises $\Delta \pi$ by the same factor while taking $c_{\mathrm{LC}}=1.5 \mathrm{mM} \mathrm{NaCl}$. That is, PRO is able to fully utilize the augmented concentration difference to produce greater water flux and, hence, generate higher PDs (Figure 4).

On the other hand, RED experiences only marginal enhancements in driving force because the Nernst potential is logarithmically dependent on solution concentrations (eq 4). In the previous example where $c_{\mathrm{HC}}$ is increased from 0.6 to $4.0 \mathrm{M}$ $\mathrm{NaCl}(\sim 6.7 \times)$, the Nernst potential is merely amplified $\sim 32 \%$. To access the larger $\Delta G_{\text {mix }}$ of the greater salinity differences, more moles of salt permeate across as counterions (horizontal axis-intercept of Figure 5B scales directly with $c_{\mathrm{HC}}$ ) and, hence, the controlled mixing process is undesirably protracted. In other words, although more energy is available with larger salinity gradients, RED power density is only slightly enhanced and more membrane area is needed to access the energy (Figure 4). Therefore, PRO is able to take advantage of the higher $\mathcal{c}_{\mathrm{HC}}$ in anthropogenic $(4.0 \mathrm{M})$ and engineered $(1.2 \mathrm{M})$ salinity gradients, whereas the principle difference in energy utilization intrinsically excludes RED from exploiting the larger salinity difference to significantly improve PD, even with high permselectivity membranes.

\section{IMPLICATIONS}

Energy from salinity gradients can be extracted with PRO and RED, two membrane-based technologies with intrinsically different principles. The analysis presented here indicates that, with existing technologically available membranes, PRO is able to achieve greater efficiency and higher power density performance for a range of salinity gradients, compared to RED. PRO is especially proficient at extracting salinity energy from large concentration differences: for equal flow rates of $\mathrm{HC}$ and LC solutions, significantly enhanced PDs can be attained while maintaining over $45 \%$ efficiency in a cocurrent flow module. The remarkable power density performance is attributed to the inherent characteristic of PRO to effectively utilize larger salinity differences for driving force augmentation. Additionally, overall PRO productivity can be optimized by suitably tuning the membrane permeability-selectivity to substantially improve power density at a small efficiency cost.

In contrast, RED is innately unable to gain appreciable power density benefits from salinity gradient increases, regardless of membrane transport properties. Furthermore, the Donnan exclusion effect, which confers selectivity to ion exchange membranes, is overwhelmed at high solution concentrations for current RED membranes, severely constraining the technology to impractically low efficiencies. These factors restrict the feasible application of RED energy production to relatively small salinity gradients. In addition to the relatively lower PD and $\eta$, ion exchange membranes employed in RED stacks are considerably more expensive than salt-rejecting polymeric membranes used in PRO modules, further handicapping the comparative advantage of RED. ${ }^{2}$ To advance RED beyond its current limitations, technological innovations are necessary to unhinge the conductivity-permselectivity trade-off and develop ion exchange membranes with simultaneously greater permselectivity and higher conductivity.

Although PRO can potentially achieve excellent performance with large salinity gradients, realization of the technology can be hindered by inadequate membrane robustness. For example, to achieve the potential high $\mathrm{PD}$ and $\eta$ for engineered salinity gradients, the polymeric thin-film composite membrane modules need to withstand hydraulic pressures $>90$ bar (1300 psi) while retaining their structural and transport properties. Presently, the highest $\Delta P$ demonstration reported in literature is $48 \mathrm{bar}(700 \mathrm{psi})$ on a coupon-sized membrane in a laboratory testing cell. ${ }^{35}$ The large pressurization necessary for high salinity operation is likely to detrimentally alter the membrane properties and, therefore, improving the membrane mechanical robustness, together with the apt design of spacer support and membrane module, will be critical. ${ }^{35,42}$

The comparison presented in this study centers on membrane-level performance. Actual power generation installations would further comprise engineering components that are different for PRO and RED. For example, PRO requires pumps, pressure exchangers, and hydroturbines to convert mechanical expansion of the $\mathrm{HC}$ draw solution to electrical energy. RED employs a reversible redox couple at the end electrodes to directly convert salinity energy to electricity without a mechanical intermediate, and requires pumping energy to circulate the solutions through narrow stack channels. Additionally, foulants present in natural and anthropogenic input streams (e.g., river water and wastewater effluent) can detrimentally lower PRO and RED productivity. ${ }^{54,55}$ Further cost-efficiency analysis of the system-level components, taking into account fouling impacts and pretreatment, are necessary to more accurately assess the practical potential of power generation from salinity gradients.

\section{ASSOCIATED CONTENT}

\section{S Supporting Information}

Details of the molar concentration approximations for PRO and RED driving force; discussion on the practical operation of PRO module and RED stack; activity coefficient of water and salt $(\mathrm{NaCl})$ as a function of mole fraction (Figure S1); osmotic pressure difference and Nernst potential, with and without simplifying assumptions, as a function of the molar concentration (Figure S2); representative plot of power density and efficiency for a range of constant applied hydraulic pressure in PRO and constant external load resistance in RED (Figure S3); $\Delta G_{\text {mix }}, \mathrm{PD}, \eta$, percent of frictional/resistive losses, uncontrolled mixing losses, and unutilized energy, $\Delta P$, and $R_{\mathrm{L}} A_{\mathrm{m}}$ for the HC-LC solution pairings of seawater-river water, brine-wastewater, and engineered solutions (Table S1). This material is available free of charge via the Internet at http:// pubs.acs.org.

\section{AUTHOR INFORMATION}

\section{Corresponding Author}

*M. Elimelech. E-mail: menachem.elimelech@yale.edu. Phone: +12034322789. 


\section{Notes}

The authors declare no competing financial interest.

\section{ACKNOWLEDGMENTS}

We acknowledge the support received from the National Science Foundation under Award Number CBET 1232619 and from the Advanced Research Projects Agency-Energy (ARPAE), U.S. Department of Energy, via Grant DE-AR0000306. We also acknowledge the Graduate Fellowship (to Ngai Yin Yip) made by the Environment and Water Industrial Development Council of Singapore.

\section{REFERENCES}

(1) Pattle, R. E. Production of electric power by mixing fresh and salt water in the hydroelectric pile. Nature 1954, 174 (4431), 660-660.

(2) Logan, B. E.; Elimelech, M. Membrane-based processes for sustainable power generation using water. Nature 2012, 488 (7411), 313-319.

(3) Yip, N. Y.; Elimelech, M. Thermodynamic and energy efficiency analysis of power generation from natural salinity gradients by pressure retarded osmosis. Environ. Sci. Technol. 2012, 46 (9), 5230-5239.

(4) Han, G.; Zhang, S.; Li, X.; Chung, T.-S. High performance thin film composite pressure retarded osmosis (PRO) membranes for renewable salinity-gradient energy generation. J. Membr. Sci. 2013, 440, $108-121$.

(5) Achilli, A.; Prante, J. L.; Hancock, N. T.; Maxwell, E. B.; Childress, A. E. Experimental results from RO-PRO: A next generation system for low-energy desalination. Environ. Sci. Technol. 2014, 48 (11), 6437-6443.

(6) Lin, S.; Yip, N. Y.; Cath, T. Y.; Osuji, C. O.; Elimelech, M. Hybrid pressure retarded osmosis-membrane distillation system for power generation from low-grade heat: Thermodynamic analysis and energy efficiency. Environ. Sci. Technol. 2014, 48 (9), 5306-5313.

(7) Loeb, S. Osmotic power-plants. Science 1975, 189 (4203), 654655.

(8) Achilli, A.; Childress, A. E. Pressure retarded osmosis: From the vision of Sidney Loeb to the first prototype installation - Review. Desalination 2010, 261 (3), 205-211.

(9) Weinstein, J. N.; Leitz, F. B. Electric-power from difference in salinity - Dialytic battery. Science 1976, 191 (4227), 557-559.

(10) Post, J. W.; Hamelers, H. V. M; Buisman, C. J. N. Energy recovery from controlled mixing salt and fresh water with a reverse electrodialysis system. Environ. Sci. Technol. 2008, 42 (15), 57855790.

(11) Brogioli, D. Extracting renewable energy from a salinity difference using a capacitor. Phys. Rev. Lett. 2009, 103 (5), 058501.

(12) La Mantia, F.; Pasta, M.; Deshazer, H. D.; Logan, B. E.; Cui, Y. Batteries for efficient energy extraction from a water salinity difference. Nano Lett. 2011, 11 (4), 1810-1813.

(13) Siria, A.; Poncharal, P.; Biance, A. L.; Fulcrand, R.; Blase, X.; Purcell, S. T.; Bocquet, L. Giant osmotic energy conversion measured in a single transmembrane boron nitride nanotube. Nature 2013, 494 (7438), 455-458.

(14) Zhu, X.; Yang, W.; Hatzell, M. C.; Logan, B. E. Energy recovery from solutions with different salinities based on swelling and shrinking of hydrogels. Environ. Sci. Technol. 2014, 48 (12), 7157-63.

(15) News Release: Progress blue energy generation by RED. http:// www.fujifilmmembranes.com/item/progress-blue-energy-generationby-red (accessed June 05).

(16) Yip, N. Y.; Elimelech, M. Performance Limiting Effects in Power Generation from Salinity Gradients by Pressure Retarded Osmosis. Environ. Sci. Technol. 2011, 45 (23), 10273-10282.

(17) Veerman, J.; de Jong, R. M.; Saakes, M.; Metz, S. J.; Harmsen, G. J. Reverse electrodialysis: Comparison of six commercial membrane pairs on the thermodynamic efficiency and power density. J. Membr. Sci. 2009, 343 (1-2), 7-15.
(18) Thorsen, T.; Holt, T. The potential for power production from salinity gradients by pressure retarded osmosis. J. Membr. Sci. 2009, 335 (1-2), 103-110.

(19) Lin, S.; Straub, A. P.; Elimelech, M. Thermodynamic limits of extractable energy by pressure retarded osmosis. Energy Environ. Sci. 2014, 7 (8), 2706.

(20) Długolęcki, P.; Gambier, A.; Nijmeijer, K.; Wessling, M. Practical potential of reverse electrodialysis as process for sustainable energy generation. Environ. Sci. Technol. 2009, 43 (17), 6888-6894.

(21) Vermaas, D. A.; Guler, E.; Saakes, M.; Nijmeijer, K. Theoretical power density from salinity gradients using reverse electrodialysis. Energy Procedia 2012, 20, 170-184.

(22) Daniilidis, A.; Herber, R.; Vermaas, D. A. Upscale potential and financial feasibility of a reverse electrodialysis power plant. Applied Energy 2014, 119, 257-265.

(23) Feinberg, B. J.; Ramon, G. Z.; Hoek, E. M. V. Thermodynamic Analysis of Osmotic Energy Recovery at a Reverse Osmosis Desalination Plant. Environ. Sci. Technol. 2013, 47 (6), 2982-2989.

(24) Post, J. W.; Veerman, J.; Hamelers, H. V. M; Euverink, G. J. W.; Metz, S. J.; Nymeijer, K.; Buisman, C. J. N. Salinity-gradient power: Evaluation of pressure-retarded osmosis and reverse electrodialysis. J. Membr. Sci. 2007, 288 (1-2), 218-230.

(25) Yip, N. Y.; Vermaas, D. A.; Nijmeijer, K.; Elimelech, M. Thermodynamic, energy efficiency, and power density analysis of reverse electrodialysis power generation with natural salinity gradients. Environ. Sci. Technol. 2014, 48 (9), 4925-4936.

(26) Cath, T. Y.; Childress, A. E.; Elimelech, M. Forward osmosis: Principles, applications, and recent developments. J. Membr. Sci. 2006, 281 (1-2), 70-87.

(27) Yip, N. Y.; Tiraferri, A.; Phillip, W. A.; Schiffrnan, J. D.; Hoover, L. A.; Kim, Y. C.; Elimelech, M. Thin-film composite pressure retarded osmosis membranes for sustainable power generation from salinity gradients. Environ. Sci. Technol. 2011, 45 (10), 4360-4369.

(28) Robinson, R. A.; Stokes, R. H. Electrolyte Solutions, 2nd rev. ed.; Dover Publications: Mineola, NY, 2002; p xv, 571.

(29) Veerman, J.; Saakes, M.; Metz, S. J.; Harmsen, G. J. Reverse electrodialysis: Evaluation of suitable electrode systems. J. Appl. Electrochem. 2010, 40 (8), 1461-1474.

(30) Veerman, J.; Saakes, M.; Metz, S. J.; Harmsen, G. J. Reverse electrodialysis: Performance of a stack with 50 cells on the mixing of sea and river water. J. Membr. Sci. 2009, 327 (1-2), 136-144.

(31) Vermaas, D. A.; Saakes, M.; Nijmeijer, K. Enhanced mixing in the diffusive boundary layer for energy generation in reverse electrodialysis. J. Membr. Sci. 2014, 453, 312-319.

(32) Pitzer, K. S.; Peiper, J. C.; Busey, R. H. Thermodynamic properties of aqueous sodium chloride solutions. J. Phys. Chem. Ref. Data 1984, 13 (1), 1-102.

(33) Lee, K. L.; Baker, R. W.; Lonsdale, H. K. Membranes for power generation by pressure-retarded osmosis. J. Membr. Sci. 1981, 8 (2), 141-171.

(34) Bui, N.-N.; McCutcheon, J. R. Nanofiber supported thin-film composite membrane for pressure-retarded osmosis. Environ. Sci. Technol. 2014, 48 (7), 4129-4136.

(35) Straub, A. P.; Yip, N. Y.; Elimelech, M. Raising the bar: Increased hydraulic pressure allows unprecedented high power densities in pressure-retarded osmosis. Environ. Sci. Technol. Lett. 2013, 1 (1), 55-59.

(36) Geise, G. M.; Park, H. B.; Sagle, A. C.; Freeman, B. D.; McGrath, J. E. Water permeability and water/salt selectivity tradeoff in polymers for desalination. J. Membr. Sci. 2011, 369 (1-2), 130-138.

(37) Elimelech, M.; Phillip, W. A. The future of seawater desalination: Energy, technology, and the environment. Science 2011, 333 (6043), 712-717.

(38) Fritzmann, C.; Löwenberg, J.; Wintgens, T.; Melin, T. State-ofthe-art of reverse osmosis desalination. Desalination 2007, 216 (1-3), $1-76$.

(39) Arena, J. T.; McCloskey, B.; Freeman, B. D.; McCutcheon, J. R. Surface modification of thin film composite membrane support layers 
with polydopamine: Enabling use of reverse osmosis membranes in pressure retarded osmosis. J. Membr. Sci. 2011, 375 (1-2), 55-62.

(40) Chou, S.; Wang, R.; Shi, L.; She, Q.; Tang, C.; Fane, A. G. Thinfilm composite hollow fiber membranes for pressure retarded osmosis (PRO) process with high power density. J. Membr. Sci. 2012, 389, 2533.

(41) Hoover, L. A.; Schiffman, J. D.; Elimelech, M. Nanofibers in thin-film composite membrane support layers: Enabling expanded application of forward and pressure retarded osmosis. Desalination 2013, 308, 73-81.

(42) She, Q.; Jin, X.; Tang, C. Y. Osmotic power production from salinity gradient resource by pressure retarded osmosis: Effects of operating conditions and reverse solute diffusion. J. Membr. Sci. 2012, 401-402, 262-273.

(43) Geise, G. M.; Hickner, M. A.; Logan, B. E. Ionic resistance and permselectivity tradeoffs in anion exchange membranes. ACS Appl. Mater. Interfaces 2013, 5 (20), 10294-10301.

(44) Guler, E.; Zhang, Y.; Saakes, M.; Nijmeijer, K. Tailor-made anion-exchange membranes for salinity gradient power generation using reverse electrodialysis. ChemSusChem 2012, 5 (11), 2262-2270.

(45) Długołęcki, P.; Nymeijer, K.; Metz, S.; Wessling, M. Current status of ion exchange membranes for power generation from salinity gradients. J. Membr. Sci. 2008, 319 (1-2), 214-222.

(46) Güler, E.; Elizen, R.; Vermaas, D. A.; Saakes, M.; Nijmeijer, K. Performance-determining membrane properties in reverse electrodialysis. J. Membr. Sci. 2013, 446, 266-276.

(47) Baker, R. W. Membrane Technology and Applications, 3rd ed.; John Wiley \& Sons: Chichester, West Sussex; Hoboken, NJ, 2012; p xiv, 575.

(48) Mulder, M. Basic Principles of Membrane Technology, 2nd ed.; Kluwer Academic: Dordrecht, The Netherlands; Boston, MA, 1996; p 564

(49) Daniilidis, A.; Vermaas, D. A.; Herber, R.; Nijmeijer, K. Experimentally obtainable energy from mixing river water, seawater or brines with reverse electrodialysis. Renewable Energy 2014, 64, 123131.

(50) Vermaas, D. A.; Saakes, M.; Nijmeijer, K. Doubled power density from salinity gradients at reduced intermembrane distance. Environ. Sci. Technol. 2011, 45 (16), 7089-7095.

(51) Skilhagen, S. E. Osmotic power - A new, renewable energy source. Desalin. Water Treat. 2010, 15 (1-3), 271-278.

(52) Post, J. W.; Goeting, C. H.; Valk, J.; Goinga, S.; Veerman, J.; Hamelers, H. V. M; Hack, P. J. F. M. Towards implementation of reverse electrodialysis for power generation from salinity gradients. Desalin. Water Treat. 2010, 16 (1-3), 182-193.

(53) Vermaas, D. A.; Veerman, J.; Yip, N. Y.; Elimelech, M.; Saakes, M.; Nijmeijer, K. High efficiency in energy generation from salinity gradients with reverse electrodialysis. ACS Sustain Chem. Eng. 2013, 1 (10), 1295-1302.

(54) Yip, N. Y.; Elimelech, M. Influence of natural organic matter fouling and osmotic backwash on pressure retarded osmosis energy production from natural salinity gradients. Environ. Sci. Technol. 2013, 47 (21), 12607-12616

(55) Vermaas, D. A.; Kunteng, D.; Saakes, M.; Nijmeijer, K. Fouling in reverse electrodialysis under natural conditions. Water Res. 2013, 47 (3), 1289-1298. 